\title{
Desigualdad socioespacial y segregación escolar en la ciudad de Lima
}

\author{
Sandra Carrillo \\ Instituto de Estudios Peruanos (IEP) \\ https://orcid.org/0000-0003-2871-4702 \\ scarrillo@iep.org.pe \\ F. Javier Murillo \\ Universidad Autónoma de Madrid (UAM) \\ https://orcid.org/0000-0002-8003-4133 \\ javier.murillo@uam.es
}

Recibido: 22/10/2021

Aprobado: 29/11/2021 


\title{
Desigualdad socioespacial y segregación escolar en la ciudad de Lima
}

\begin{abstract}
Resumen
Esta investigación profundiza en la relación entre la desigualdad socioespacial y la segregación escolar centrándose en Lima Metropolitana. Para explorar dicha relación, en primer lugar, se busca estimar la segregación escolar en el nivel de educación primaria en cada distrito y su vínculo con su nivel económico, así como el aporte diferencial de las escuelas estatales y no estatales. En segundo lugar, se describe la variación de la segregación escolar según la distribución socioespacial en tres distritos. En función de ello, se realiza una explotación especial de los datos de la Evaluación Censal de Estudiantes (ECE) del 2018 del Ministerio de Educación y se utiliza el índice de raíz cuadrada de Hutchens. Se analiza datos correspondientes a 113.233 estudiantes de $4^{\circ}$ grado de educación primaria, distribuidos en 3859 servicios educativos situados en 41 distritos de Lima Metropolitana. Mediante dicho análisis, se encontró una fuerte relación entre la segregación socioespacial y la segregación escolar, especialmente, la segregación de los estudiantes más vulnerables, así como el importante peso de la educación no estatal en esta relación. Sin embargo, también, se identificó que la desigualdad socioespacial no determina la segregación: hay distritos que tienen una segregación baja independientemente de sus características socioeconómicas. Estos datos llevan a concluir la necesidad de implementar medidas de política educativa pública contra la segregación, que combinen marcos regulatorios comunes con intervenciones diferenciadas en distintos territorios en función de sus características. Una de esas medidas debe afectar a la educación no estatal en diferentes ámbitos.
\end{abstract}

Palabras clave: Desigualdad socioespacial, educación básica, nivel socioeconómico, Perú, segregación escolar. 


\title{
Socio-spatial inequality and school segregation in the city of Lima
}

\begin{abstract}
This research analyses the relationship between socio-spatial inequality and school segregation, focusing on Metropolitan Lima. First, we estimate school segregation at the primary school level in each district and its relationship with its economic level, as well as the differential contribution of state and private schools. Second, we describe the variation of school segregation according to the socio-spatial distribution of three districts. For this purpose, the study serves from special data mining of the 2018 National Student Assessment (ECE) data from the Ministry of Education an applies the Hutchens Square Root index. We analyse data from 113.233 students in 4th grade of Primary Education, distributed in 3859 schools located in 41 districts of Metropolitan Lima. Research shows a strong relationship between socio-spatial segregation and school segregation, specially segregating the most vulnerable students, as well as the important weight of private education in this relationship. However, it also demonstrates that socio-spatial inequality does not determine segregation: there are districts that have low segregation regardless of their socioeconomic characteristics. This information leads to the conclusion that there is a need to implement public education policy measures against segregation, combining common regulatory frameworks with differentiated interventions in different territories according to their characteristics. One of these measures should affect private education in different areas.
\end{abstract}

Keywords: Socio-spatial inequality, basic education, socioeconomic level, Peru, school segregation 


\section{Introducción ${ }^{1}$}

Recibir una educación de calidad es un derecho humano básico de todas las personas, y, como tal, las administraciones públicas tienen la obligación de asegurar, y la sociedad de exigir su cumplimiento y colaborar con su consecución. En esa lógica, el acceso a la educación, una enseñanza de calidad y garantizar la participación, y el pleno desarrollo de todos debe constituirse como la máxima obligación de los poderes públicos, sin importar dónde se haya nacido, la riqueza de sus familias, sus capacidades, su lengua materna, su pertenencia a grupo cultural o su religión. No caben excepciones cuando se trata del derecho a recibir una educación de calidad.

Sin embargo, la igualdad de oportunidades se pone en entredicho cuando existe una distribución no aleatoria de los estudiantes en diferentes escuelas según sus características personales o condiciones sociales, lo que hace que las escuelas sean homogéneas hacia adentro (en relación con estas características o condiciones) y muy heterogéneas entre sí. Este fenómeno, técnicamente conocido como segregación escolar, atenta directamente contra ese derecho.

En esta discusión global y sobre la base de estudios comparados, el Perú es uno de los países de América Latina con una mayor segregación de estudiantes en diferentes escuelas en función del nivel socioeconómico de las familias, medida que se ha ido incrementando en los últimos años, tanto para el nivel de primaria (Murillo, 2016; Murillo y Martínez-Garrido, 2017a) como para el de secundaria (Benavides et al., 2014; Krüger, 2019; Murillo et al., 2018; OECD, 2019; Vazquez, 2016). En los estudios desarrollados en Perú, se identifica una alta segregación escolar por nivel socioeconómico, pero con una gran variabilidad entre sus departamentos; los estudiantes de menor nivel socioeconómico son aquellos que sufren mayor segregación (Murillo y Carrillo, 2020a, 2000b).

Para aportar a este debate, la presente investigación pretende profundizar en la relación entre la desigualdad socioespacial y la segregación escolar a través del estudio de una ciudad altamente desigual y con una alta segregación escolar, como es Lima Metropolitana.

\section{Marco teórico}

La segregación escolar es un fenómeno complejo y de creciente interés en la región. Como se ha mencionado, y según la definición de Dupriez (2010), esta se refiere a la distribución desigual o desbalanceada de los alumnos en los centros educativos, según sus características personales o condiciones sociales. La característica principal se vincula con la desventaja que sufren los grupos minoritarios que se encuentran segregados en relación con el grupo mayoritario.

1. Este artículo forma parte de una línea de investigación desarrollada por ambos autores sobre la segregación escolar en el Perú en el marco del Programa de Doctorado en Educación de la Universidad Autónoma de Madrid. 
La segregación escolar por nivel socioeconómico es la que más afecta a América Latina y, a su vez, la que más incide en la inequidad social (Murillo, 2016). Esta se refiere a la distribución no aleatoria de los estudiantes entre las escuelas según el nivel de ingresos y/o recursos de sus familias. Es decir, un sistema educativo con altos niveles de segregación tiene instituciones educativas muy diferenciadas entre sí, pero cuyo estudiantado es bastante homogéneo en relación con, en este caso, su nivel socioeconómico.

La segregación escolar puede medirse desde dos dimensiones. Como señala Murillo (2016), una se refiere al grado de la distribución de los estudiantes - en este caso, según el nivel socioeconómico de sus familias- en las diferentes escuelas. Es decir, si la distribución de un grupo es muy desigual entre las escuelas, identificamos que se encuentra segregado en su dimensión de igualdad o uniformidad. No obstante, si hablamos de bajas probabilidades de interacción entre los estudiantes de diferentes niveles socioeconómicos, nos referimos a la dimensión de exposición, es decir, el grado de aislamiento relativo de un grupo segregado/ minoritario respecto a otros.

Ambas dimensiones son complementarias para explicar la segregación escolar y existen diferentes índices para medirlas. Así, para estimar la segregación escolar en su dimensión de uniformidad, los índices más habituales son los índices de Duncan, la raíz cuadrada de Hutchens y el índice de Gorard, cada uno de los cuales tiene sus propias características. De manera complementaria, en su dimensión de exposición, los índices más comunes son el índice de aislamiento y el de interacción mutua (Murillo et al., 2018).

Las causas de la segregación son múltiples y complejas; estas pueden responder tanto a factores propios de los sistemas educativos como a factores externos a ellos. Al respecto, Natalia Krüger (2019) plantea que, si bien se han identificado algunos elementos comunes a los sistemas educativos, marcados por las tendencias globales, muchos de ellos dependen de los propios contextos en los que se desarrollan (como, por ejemplo, normas, políticas y actores de cada país). Por su parte, para Cristián Bellei (2013), la segregación escolar es una consecuencia agregada de acciones que, en muchos casos, han sido indeseadas o no anticipadas. Así, por ejemplo, uno de los factores endógenos estudiados, y que se relacionan con la segregación escolar, es la configuración de los mercados educativos (Alegre, 2010). A ello se suman las particularidades de cada país. Un ejemplo es el caso de Chile, sobre el cual existen abundantes estudios que tratan de explicar cómo el financiamiento compartido profundiza la segregación escolar (Bellei et al., 2018; Elacqua, 2009; Santos y Elacqua, 2016; Valenzuela et al., 2014).

Otro de los factores estudiados como un elemento exógeno a los sistemas educativos es la segregación residencial. Los primeros estudios planteaban que la segregación escolar respondía a una configuración "natural" de los territorios y que, por lo tanto, el problema de fondo era la distribución socioespacial de la población más que un problema del sistema educativo (Martínez y Ferrer, 2018). Sin embargo, para investigadores como Sabatini (1999), explicar la 
segregación escolar únicamente desde la segregación residencial es muy peligroso, dado que se invisibiliza las dinámicas que hay detrás de este fenómeno y no se aborda el problema de manera directa.

Las consecuencias de la segregación vienen siendo ampliamente estudiadas y pueden organizarse en tres grandes grupos. El primero refiere a los aspectos de formación ciudadana que ofrece el sistema educativo, los que en el mediano y largo plazo se vinculan con los aspectos de la convivencia e integración social (Bellei, 2013; García-Huidobro, 2007, Miranda, 2021). Transitar a lo largo de la educación básica entre estudiantes con características o condiciones sociales muy parecidas entre sí conduce a que se pierda la oportunidad de la experiencia de aprender a convivir en la diversidad. El segundo grupo se relaciona con el efecto de la segregación en la calidad de los aprendizajes, principalmente, en los grupos más vulnerables. $\mathrm{Al}$ respecto, existe amplia literatura sobre el efecto de pares y cómo los grupos con mayores dificultades para el aprendizaje se benefician en aulas diversas, sobre todo, con estudiantes con mejores aprendizajes y mayores recursos (Burke y Sass, 2013; Cueto et al., 2016; Murillo y Carrillo, 2021). El tercer grupo corresponde a la implementación de las políticas educativas y su efectividad, dado que la segregación tiene un efecto colectivo sobre la exclusión social. En el mediano y largo plazo, este fenómeno refuerza las desigualdades persistentes y trampas de pobreza, por lo que complejiza y hace más costosa la intervención sobre los grupos más desfavorecidos (Durlauf, 2006; Valenzuela et al., 2014).

Como hemos señalado previamente, el Perú es uno de los países de la región con la más alta segregación escolar por nivel socioeconómico. Si bien el país cuenta con un conjunto importante de estudios sobre las brechas y desigualdades en el sistema educativo (Cuenca y Urrutia, 2019; Cueto, 2021; Guadalupe et al., 2017), los estudios sobre segregación escolar son recientes. Investigaciones como la de Benavides et al. (2014) muestran cómo la segregación escolar se ha incrementado entre los años 2000 y 2009, y plantean como hipótesis que esto podría deberse al incremento de la oferta educativa privada. Balarin y Escudero (2018) manejan esta misma hipótesis, y atribuyen dicho fenómeno de manera más específica a las escuelas de bajo costo. Otro grupo de estudios trata de describir su magnitud en los diferentes niveles y regiones del país (Murillo y Carrillo, 2020a, 2020b) y su incidencia en el rendimiento de los estudiantes (Murillo y Carrillo, 2021).

Complementariamente, se encuentra una serie de estudios sobre Lima Metropolitana, ciudad caracterizada por una marcada estratificación social, que refleja las desigualdades entre su población. Una proporción importante de estudiantes de educación básica del país se ubica en este territorio y es una de las ciudades con mayor incremento de la oferta privada en las últimas décadas (Carrillo et al., 2019; Cuenca, 2013; Ministerio de Educación, 2018b).

La segregación socioespacial de Lima se acentúa más por las diferencias socioeconómicas que por las diferencias étnicas, como sucede en la mayoría de las ciudades de América Latina (Roca, 2012). Ello se expresa principalmente en un 
patrón residencial por el cual la población de menores recursos se ubica mayoritariamente en las zonas periféricas (espacios urbanos en proceso de consolidación) y la población con mayores recursos se asienta principalmente en espacios urbanos consolidados. De esta manera, se puede identificar claramente espacios ocupados, casi de manera exclusiva, por poblaciones de las mismas características que habitan la ciudad de manera próxima, lo que a su vez muestra la forma como el espacio urbano es configurado de acuerdo con las inequidades y el proceso de exclusión característicos de la sociedad peruana (Pereyra 2006; Wiese et al., 2016).

Estas diferencias socioespaciales se mantienen en la oferta educativa de la educación primaria en Lima Metropolitana. El Ministerio de Educación (2018b) identifica la oferta de escuelas privadas de alto y muy alto costo (pensión mayor a 970 soles) en las áreas con mayor nivel de consolidación urbana, y los centros educativos no estatales y de bajo costo (pensión de 291 soles a menos) en los distritos de menor nivel socioeconómico. Además, las escuelas de primaria más caras, así como las escuelas estatales ubicadas en los distritos de mayor nivel socioeconómico, son las que suelen tener mejores puntajes en la Evaluación Censal de Estudiantes (ECE) (Ministerio de Educación, 2018b).

Con esta investigación, se pretende indagar en la relación entre la desigualdad socioespacial y la segregación escolar centrándonos en Lima Metropolitana. Para explorar dicha relación, en primer lugar, se busca estimar la segregación escolar en el nivel de primaria en cada distrito y su vínculo con su nivel económico, así como el aporte diferencial de las escuelas estatales y no estatales. En segundo lugar, se describe la variación de la segregación escolar según la distribución socioespacial de tres distritos seleccionados.

\section{Método}

Para desarrollar estos objetivos, se realiza una explotación de los microdatos de la Evaluación Censal de Estudiantes (ECE) correspondiente al año 2018. La ECE es la evaluación estandarizada del Ministerio de Educación de Perú, cuya finalidad es producir información sobre el desempeño de los estudiantes e identificar los niveles de logro alcanzados en diferentes áreas y grados según los niveles educativos.

Así, para este estudio, se utiliza información de 113.233 estudiantes de $4^{\circ}$ de primaria, escolarizados en 3859 servicios educativos - en adelante, para facilitar la lectura, serán denominados "escuelas" - , situados en los 41 distritos de Lima Metropolitana (Tabla 1). Para efectos del análisis, los 41 distritos se han agrupado en cuatro zonas, según la ubicación socioespacial: Lima Centro, Lima Norte, Lima Sur y Lima Este².

2. Como se señala en Carrillo et al. (2019), la división entre los distritos de la periferia (Lima Norte, Lima Este, Lima Sur) y Lima Centro suele ser la más común y difundida. Se usa, por ejemplo, en los estudios urbanísticos (niveles de consolidación urbana), de opinión pública e investigación de mercado, por nombrar algunos casos. 
Tabla 1

Estudiantes y servicios educativos (escuelas) participantes en el estudio

\begin{tabular}{|c|c|c|c|c|}
\hline Distrito & $\begin{array}{c}\text { Número } \\
\text { de estudiantes }\end{array}$ & $\begin{array}{c}\text { Número } \\
\text { de servicios } \\
\text { educativos }\end{array}$ & $\begin{array}{c}\% \text { servicios } \\
\text { educativos } \\
\text { estatales }\end{array}$ & $\begin{array}{l}\text { Tasa de } \\
\text { pobreza }\end{array}$ \\
\hline \multicolumn{5}{|l|}{ Lima Norte } \\
\hline Ancón & 863 & 33 & 36,36 & 13,0 \\
\hline Carabayllo & 4274 & 156 & 19,87 & 15,0 \\
\hline Comas & 6602 & 236 & 26,69 & 12,9 \\
\hline Independencia & 2285 & 74 & 31,08 & 26,0 \\
\hline Los Olivos & 5387 & 185 & 18,38 & 6,3 \\
\hline Puente Piedra & 4503 & 177 & 22,03 & 17,4 \\
\hline San Martin de Porres & 7573 & 350 & 18,00 & 8,6 \\
\hline Santa Rosa & 216 & 13 & 46,15 & 15,1 \\
\hline \multicolumn{5}{|l|}{ Lima Centro } \\
\hline Barranco & 940 & 23 & 43,48 & 1,6 \\
\hline Breña & 1198 & 40 & 25,00 & 2,6 \\
\hline Jesús María & 792 & 23 & 8,70 & 0,6 \\
\hline La Victoria & 2033 & 69 & 31,88 & 8,9 \\
\hline Lima & 3453 & 122 & 40,16 & 6,4 \\
\hline Lince & 498 & 18 & 16,67 & 1,5 \\
\hline Magdalena del Mar & 603 & 21 & 28,57 & 0,8 \\
\hline Miraflores & 1365 & 27 & 18,52 & 0,1 \\
\hline Pueblo Libre & 891 & 27 & 25,93 & 0,8 \\
\hline Rímac & 2112 & 68 & 35,29 & 9,1 \\
\hline San Borja & 819 & 22 & 22,73 & 0,5 \\
\hline San Isidro & 862 & 19 & 10,53 & 0,1 \\
\hline San Miguel & 1499 & 61 & 11,48 & 1,6 \\
\hline Santiago de Surco & 3364 & 100 & 13,00 & 1,2 \\
\hline Surquillo & 646 & 23 & 34,78 & 2,8 \\
\hline \multicolumn{5}{|l|}{ Lima Este } \\
\hline Ate Vitarte & 8909 & 307 & 20,20 & 14,1 \\
\hline Chaclacayo & 715 & 29 & 27,59 & 6,2 \\
\hline Cieneguilla & 310 & 13 & 46,15 & 11,8 \\
\hline El Agustino & 2263 & 69 & 40,58 & 11,8 \\
\hline La Molina & 2208 & 61 & 16,39 & 1,2 \\
\hline Lurigancho & 3206 & 118 & 35,59 & 14,1 \\
\hline San Juan de Lurigancho & 14.476 & 440 & 21,14 & 15,4 \\
\hline San Luis & 812 & 24 & 33,33 & 3,1 \\
\hline Santa Anita & 3063 & 91 & 23,08 & 6,5 \\
\hline
\end{tabular}




\begin{tabular}{lcccc}
\hline Lima Sur & & & & \\
\hline Chorrillos & 4899 & 141 & 21,99 & 11,6 \\
Lurín & 1214 & 45 & 33,33 & 14,4 \\
Pachacamac & 1851 & 54 & 33,33 & 20,0 \\
Pucusana & 183 & 8 & 37,50 & 23,8 \\
Punta Negra & 103 & 7 & 14,29 & 8,7 \\
San Bartolo & 110 & 4 & 25,00 & 8,3 \\
San Juan de Miraflores & 4850 & 169 & 26,04 & 12,0 \\
Villa el Salvador & 5380 & 190 & 22,63 & 16,6 \\
Villa María del Triunfo & 5903 & 202 & 20,79 & 13,7 \\
\hline Total Lima & 113.233 & 3.859 & 23,84 & 8,9 \\
Metropolitana & & & & \\
\hline
\end{tabular}

Nota: No se consideraron los distritos de Santa María del Mar y Punta Hermosa por contar con muy pocos servicios educativos para el análisis. Elaboración propia a partir de la base de datos ECE 2018 (Minedu-UMC, 2018) e información del INEI (2020).

Para la primera parte del estudio, se utilizó cuatro variables: el distrito en el que está ubicada la escuela, la tasa de pobreza de cada distrito como un proxy de su nivel económico (INEI, 2020), el tipo de gestión de la escuela (estatal y no estatal) y el índice socioeconómico (ISE) de la familia del estudiante. Esta última es la variable criterio utilizada para medir la segregación escolar. Se presenta tipificada (media 0 y desviación típica 1 ) y es estimada por el Ministerio de Educación a partir de variables sobre la educación de los padres, materiales de construcción de la vivienda, servicios básicos en el hogar, activos en el hogar y otros servicios en el hogar (Ministerio de Educación, 2018a).

Para la segunda parte del estudio, que consiste en describir la variación de la segregación escolar según la distribución socioespacial, se seleccionaron tres distritos: La Molina, Santa Anita e Independencia. Estos representan tres modelos de distritos en relación con dos variables: su renta per cápita (proxy de su nivel económico del distrito) y la magnitud de su segregación escolar. Además, se buscaron distritos con un número de escuelas intermedio, de entre 50 y 100, para analizar con más detalle cada escuela y comparar los hallazgos.

Se utiliza el índice de la raíz cuadrada o índice de Hutchens (IH) para estimar la segregación escolar por origen socioeconómico. Este índice tiene la propiedad de descomposición aditiva, lo que le permite determinar el aporte de varios subconjuntos a la segregación total. La estimación se realiza a partir de la suma del alejamiento de cada escuela de la igualdad distributiva, es decir, la distancia entre la media geométrica de las participaciones de estudiantes de distinto nivel socioeconómico en ausencia de segregación y la media geométrica de las participaciones reales (Hutchens, 2004). Su fórmula matemática es la siguiente: 


$$
H=\sum_{i=1}^{k}\left(\left(\frac{x_{1 i}}{X_{1}}\right)-\sqrt{\frac{x_{2 i}}{X_{2}} \frac{x_{1 i}}{X_{1}}}\right)
$$

En esta, $\mathrm{x}_{1 \mathrm{i}} \mathrm{y}_{2 \mathrm{i}}$ representan el número de alumnos del grupo minoritario y mayoritario para Lima o cada uno de sus distritos, respectivamente en la escuela i. $\mathrm{X}_{1}$ y $\mathrm{X}_{2}$ corresponden al número total de estudiantes de los grupos minoritario y mayoritario en todas las escuelas de la ciudad o distrito.

Por su propiedad de descomposición aditiva, es posible dividir el índice en dos partes: la magnitud de la segregación para cada subsistema (intra-subsistema) y la segregación generada entre ambos conjuntamente (inter-subsistemas). El componente " $\mathrm{H}$ intra-subsistema" es una suma ponderada de la segregación dentro de cada sector g. Por su parte, el componente "H inter-susbistemas" puede expresarse como fracción de $\mathrm{H}$ para evaluar qué proporción de la segregación total se debe a la distribución desigual por origen entre un subsistema y otro, en este caso, entre escuelas estatales y no estatales.

$$
H=H_{\text {intra }}+H_{\text {inter }}
$$

En esta fórmula,

$$
H_{\text {intra }}=\sum_{g=1}^{G} w_{g} H_{g}
$$

Con,

$$
w_{g}=\sqrt{\left(\frac{P_{g}}{P}\right)\left(\frac{R_{g}}{R}\right)}
$$

Donde $\mathrm{g}=1, \ldots, \mathrm{G}$ subgrupos, $\mathrm{y} \mathrm{w}_{\mathrm{g}}$ es el peso del subgrupo $\mathrm{g}, \mathrm{P}_{\mathrm{g}} \mathrm{y} \mathrm{R}_{\mathrm{g}} \mathrm{el}$ número de estudiantes en el subgrupo $\mathrm{g}$ con respecto al grupo minoritario $\mathrm{y}$ mayoritario P y R.

Para tener una imagen más completa de la segregación escolar por nivel socioeconómico, se considera dos grupos minoritarios: el 25\% de los estudiantes con familias de menor nivel socioeconómico (Q1) y el 25\% de los estudiantes con familias de mayor nivel socioeconómico (Q4). De esta manera, se estima la composición de la varianza para cada uno de los distritos de la ciudad. 


\section{Resultados}

En coherencia con los objetivos planteados, los resultados se organizan en dos apartados. En primer lugar, se presenta información general sobre la segregación escolar por nivel socioeconómico en Lima Metropolitana y sus distritos. En un segundo apartado, se realiza un estudio en profundidad de la segregación escolar en tres distritos de la ciudad capital.

\section{La segregación escolar en los distritos de Lima Metropolitana}

Lima es una ciudad con una alta segregación escolar por nivel socioeconómico, especialmente, para el $25 \%$ de estudiantes con mayores recursos económicos (Q4), que alcanza una magnitud de 0,33 en el índice H. Para el 25\% de los estudiantes con familias de menos recursos (Q1), la segregación alcanza el 0,25. En otras palabras, una tercera parte de los estudiantes de mayor nivel socioeconómico y una cuarte parte de aquellos de menor nivel socioeconómico tendrían que cambiarse de escuela para que no haya segregación o esta sea menor. Estas cifras, sin embargo, no revelan la impresionante variabilidad entre los distritos. Además, la magnitud de la segregación escolar para los estudiantes más pobres $(\mathrm{Q} 1)$ y más ricos $(\mathrm{Q} 4)$ es muy diferente en cada distrito, aspecto que será estudiado con más detalle.

La segregación escolar para el $25 \%$ de los estudiantes con familias con menos recursos (Q1) en cada distrito varía de un 0,62 en el distrito de San Isidro (Lima Centro) a un 0,08 en San Bartolo (Lima Sur). En estas diferencias, la situación geográfica de los distritos cumple un rol determinante (Gráfico 1). Así, de los 19 distritos más segregados de Lima, 15 son de Lima Centro; las únicas excepciones son los distritos de La Molina y Santa Rosa, que también presentan una alta segregación escolar, y Chorrillos y Punta Negra, con una segregación intermedia. Destaca la segregación escolar de San Isidro, con un IH de 0,62; seguido de Santiago de Surco, con 0,54; el mencionado distrito de La Molina, con 0,53; Miraflores, con 0,52; y San Miguel, con 0,51. Ello significa que la mitad de los estudiantes de menor nivel socioeconómico que estudian en estos distritos tendrían que cambiarse de escuela para disminuir la segregación.

Los distritos con una menor segregación para los estudiantes más pobres se reparten en las otras tres zonas en las que se divide Lima. El distrito menos segregado para este colectivo es San Bartolo (Lima Sur), con 0,08; Cieneguilla (Lima Este), con 0,09; Pucusana (Lima Sur), también con 0,09; e Independencia y Puente Piedra (Lima Norte), ambos con 0,13 . En estos casos, la distribución de los estudiantes es más homogénea en sus niveles socioeconómicos, por lo que no se identifican desbalances importantes en la distribución de alumnos entre las escuelas de estos distritos. 
Gráfico 1

Segregación escolar por nivel socioeconómico para Q1 en cada distrito de Lima

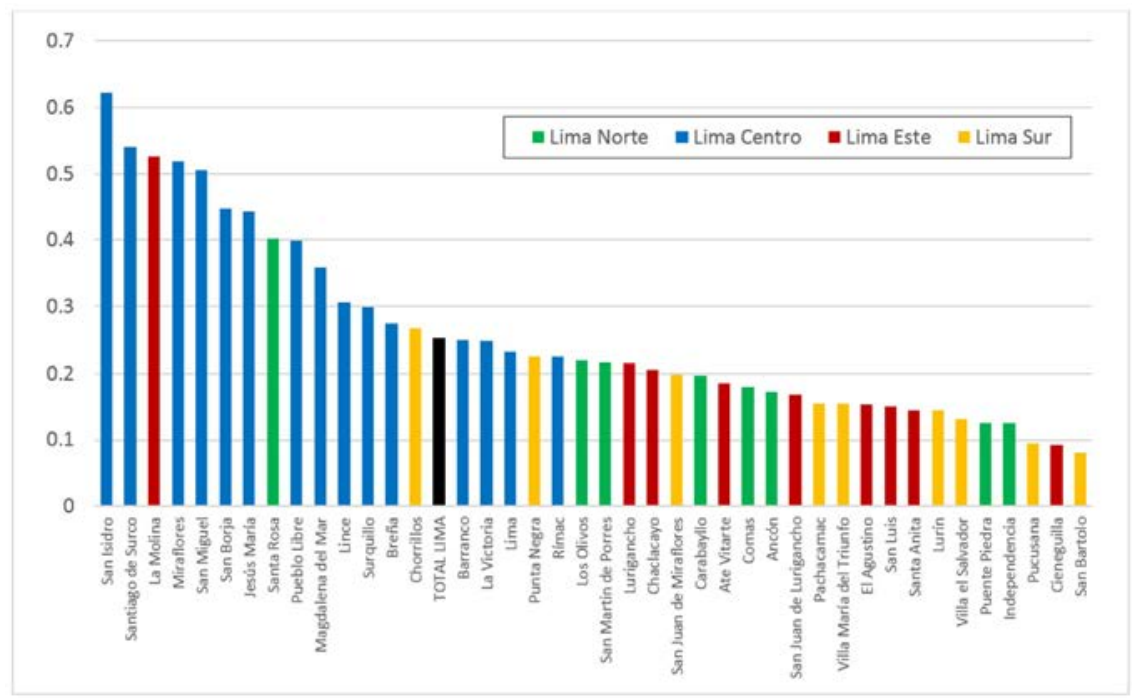

Nota: Distritos ordenados por la magnitud de la segregación. Elaboración propia a partir de la base de datos ECE 2018 (Minedu-UMC, 2018).

La segregación escolar para el $25 \%$ de estudiantes de familias con mayor nivel socioeconómico (Q4) ofrece una realidad radicalmente diferente (Gráfico 2), dado que la zona no determina la magnitud de la segregación escolar. Así, los cuatro distritos más segregados para los estudiantes con más recursos se ubican en zonas diferentes. El más segregado es Santa Rosa (Lima Norte), con un IH de 0,49; seguido de La Molina (Lima Este), con 0,49; Pachacamac (Lima Sur), con 0,46; y Miraflores (Lima Centro), con 0,44. Es decir, cinco de cada diez estudiantes con mayor nivel socioeconómico de estos distritos deberían cambiarse de escuela para disminuir los niveles de segregación. Los casos de distritos como San Bartolo (Lima Sur), con 0,11, y Breña y Madalena del Mar (ambas de Lima Centro), con una segregación de 0,16 y 0,17, respectivamente, tienen una distribución de los estudiantes según el nivel socioeconómico de sus familias un poco más balanceada, por lo que la segregación es mucho menor. 
Gráfico 2

Segregación escolar por nivel socioeconómico para Q4 en cada distrito de Lima

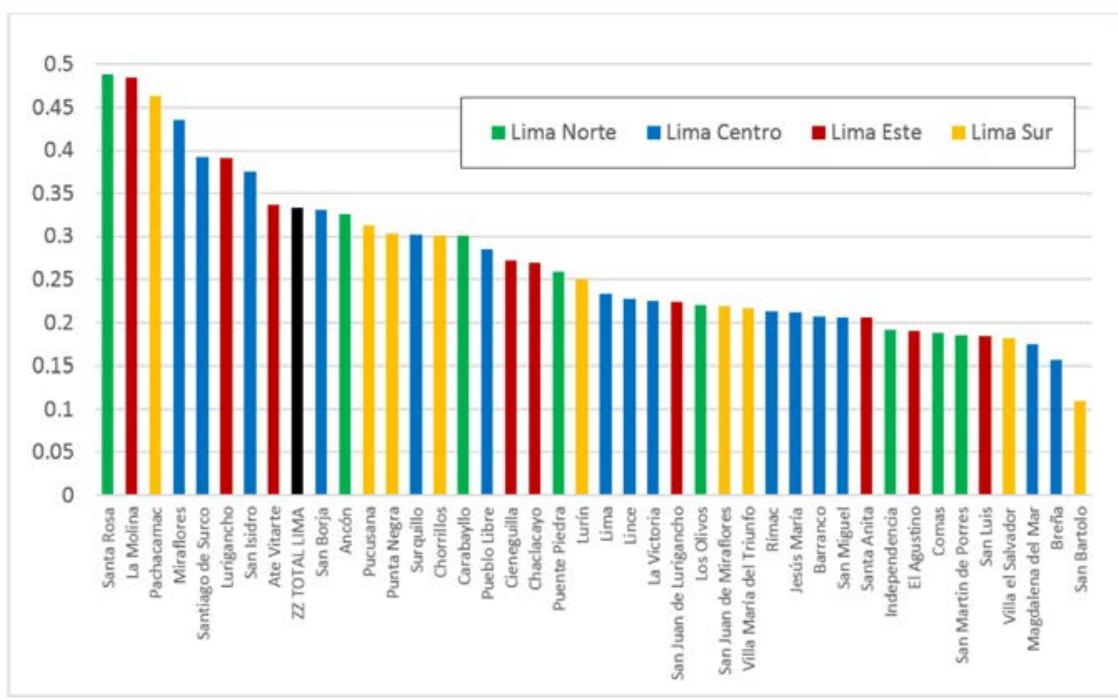

Nota: Distritos ordenados por la magnitud de la segregación. Elaboración propia a partir de la base de datos ECE 2018 (Minedu-UMC, 2018).

Como señalamos, la relación entre la segregación escolar para el Q1 y para el Q4 en cada distrito tiene un índice de correlación de 0,47. Ello implica que, aunque lo habitual es que los distritos con una mayor segregación para Q1 también lo tengan para Q4, la segregación escolar en cada distrito para los estudiantes más pobres no está definida por la segregación para los estudiantes de familias con más recursos, ni viceversa. Así, como muestra el gráfico 3, es posible encontrar distritos con las siguientes características:

- Alta segregación para Q1 y Q4: San Isidro, la Molina, Santiago de Surco y Miraflores son buenos ejemplos. La mayoría de los distritos con esta situación se ubican en Lima Centro.

- Baja segregación para Q1 y para Q4: El distrito de San Bartolo (Lima Sur) es el mejor ejemplo, aunque solo tiene cuatro escuelas. Villa el Salvador o Independencia también se encuentran en esta situación.

- Alta segregación para Q1 y baja para Q4: Los cinco distritos que se encuentran en esta situación son de Lima Centro, y resalta el distrito de San Miguel.

- Baja segregación para Q1 y alta para Q4: En este grupo, destacan Pachacamac, con muy alta segregación para Q4, y Pucusana, con muy baja en Q1. 


\section{Gráfico 3}

Relación entre la segregación escolar para los estudiantes más pobres (Q1)

y para estudiantes más ricos (Q4)

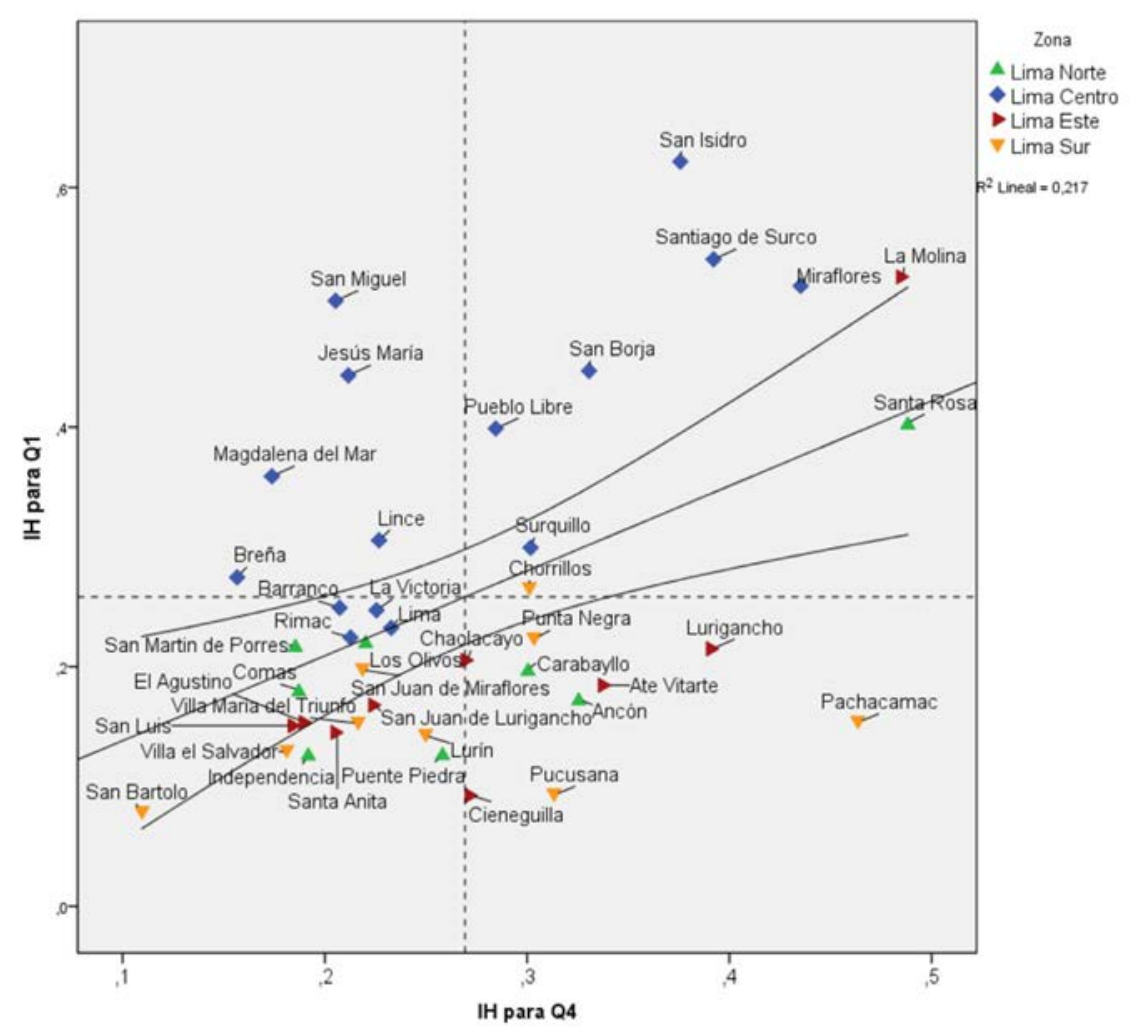

Fuente: Elaboración propia a partir de la base de datos ECE 2018 (Minedu-UMC, 2018).

La relación entre la zona de Lima en la que se ubica cada distrito y su segregación escolar para Q1 como grupo minoritario es más alta en comparación con la segregación para Q4. Ello nos hace pensar que la relación entre nivel socioeconómico del distrito y la magnitud segregación escolar se limita a la segregación del alumnado más vulnerable.

El índice de correlación entre la segregación escolar para Q1 como grupo minoritario y tasa de pobreza del distrito (proxy del nivel económico) es de $-0,70$, lo que muestra la fuerte relación entre ambas variables. Así, cuanto más pobre es un distrito, la segregación escolar para los estudiantes más vulnerables será menor. En el siguiente gráfico, se observa dicha relación. 


\section{Gráfico 4}

Relación entre la segregación escolar para los estudiantes más pobres (Q1)

y la tasa de pobreza en cada distrito

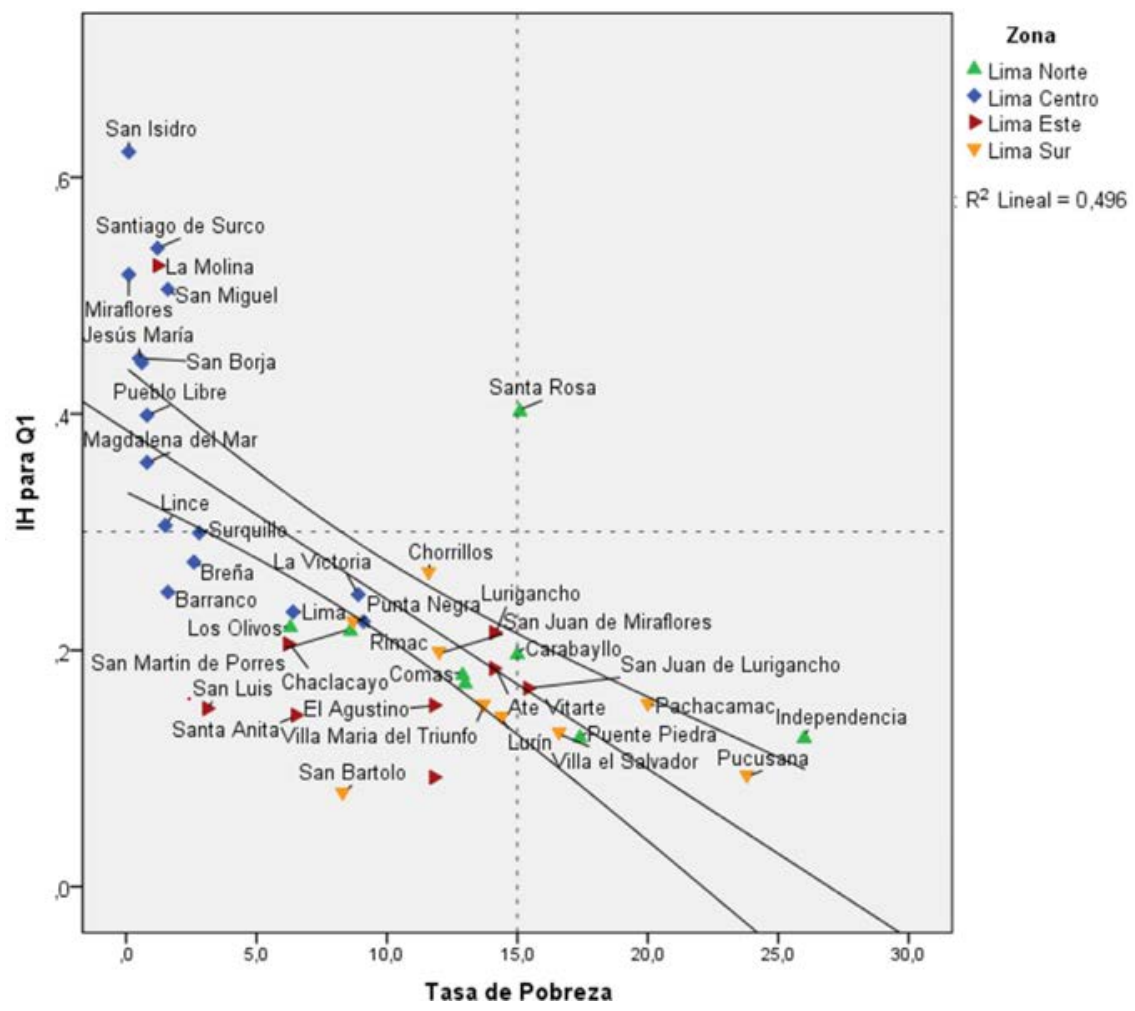

Fuente: Elaboración propia a partir de la base de datos ECE 2018 (Minedu-UMC, 2018).

La correlación entre la segregación escolar para los estudiantes con más recursos y la tasa de pobreza, sin embargo, es estadísticamente no significativa $\left(r_{x y}=0,02\right)$. Es decir, no hay una relación importante entre el nivel socioeconómico del distrito y la segregación escolar del estudiantado con mayores recursos. De esta forma, se observa que el nivel socioeconómico del distrito determina en gran medida la segregación escolar, pero solo en el caso del estudiantado con menos recursos. En esa medida, no existe una relación directa entre la pobreza de un distrito y la segregación escolar de los estudiantes de las familias más adineradas.

El análisis de la segregación diferenciada de escuelas estatales y no estatales es muy relevante para entender la segregación escolar en los distritos de la ciudad de Lima. Así, si profundizamos en el análisis de la segregación escolar por nivel socioeconómico en escuelas de gestión estatal y no estatal, 
y nos enfocamos en cada uno de los distritos de Lima Metropolitana (Tabla 5 , en anexo), encontramos una gran diferencia en la segregación escolar por nivel socioeconómico entre cada uno de ellos, que se acentúan con el nivel de recursos de las familias y el distrito al que se hace referencia.

A partir de ello, se observa que la mayor segregación escolar para los estudiantes con menos recursos (Q1) (Gráfico 5) ocurre en las escuelas de gestión privada. En este caso, los cinco distritos más segregados para los estudiantes con menos recursos se ubican en zonas diferentes: los dos más segregados son los distritos de Pueblo Libre y San Borja (ambos con IH 0,82), ubicados en Lima Centro; seguidos de Santiago de Surco ( $\mathrm{IH} 0,75)$ y Miraflores $(0,71)$, también de Lima Centro; y La Molina, distrito que representa la excepción al encontrarse en Lima Este (IH 0,72). En el caso de la oferta estatal, la segregación es menor. Los distritos más segregados son Santa Rosa (IH 0,32) en Lima Norte; La Molina (IH 0,20) en Lima Norte; y San Miguel y Santiago de Surco (con IH 019 y IH 0,18, respectivamente), ambos en Lima Centro. Sin embargo, se debe anotar que la segregación en estos casos se presenta en menor medida que en las instituciones educativas privadas (Tabla 5 en anexo).

\section{Gráfico 5}

Segregación escolar por nivel socioeconómico para Q1 en servicios educativos estatales y no estatales en cada distrito de Lima

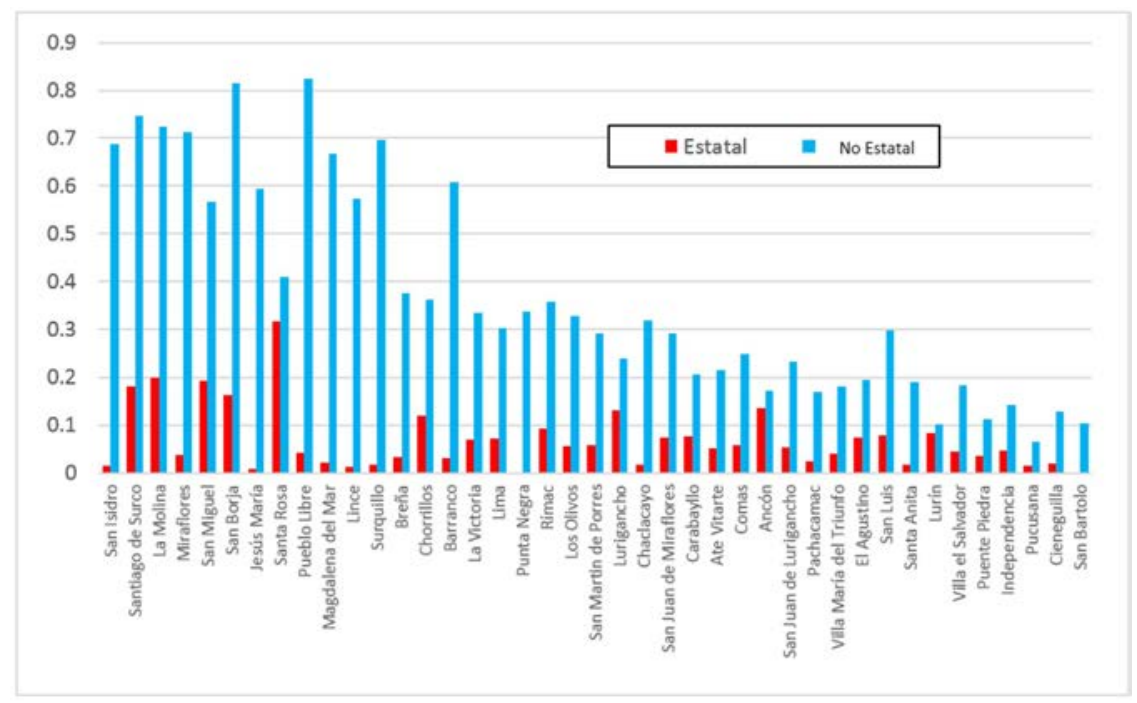

Nota: Distritos ordenados por la magnitud de la segregación total. Elaboración propia a partir de la base de datos ECE 2018 (Minedu-UMC, 2018). 
Por su parte, en el grupo de los estudiantes de familias con más recursos (Q4), la realidad es muy diferente (Gráfico 6). En este caso, la segregación escolar es mayor en la oferta de gestión estatal. Así, los cuatro distritos más segregados para los estudiantes con más recursos se encuentran en zonas diferentes. El más segregado es Santa Rosa (IH de 0,64) de Lima Norte, seguido de Pachacamac $(0,35)$ de Lima Sur y La Molina $(0,33)$ de Lima Este. En el caso de la oferta privada, los distritos San Bartolo y Punta Negra, ambos de Lima Sur, son los más segregados junto con Miraflores, de Lima Centro. Cada una cuenta con un IH de 0,37. Estos distritos son seguidos de Pachacamac (Lima Sur) y Santiago de Surco (Lima Centro) ambos con 0,34; y San Juan de Lurigancho y La Molina (Lima Este), ambos con 0,33 (Tabla 5 en anexo).

\section{Gráfico 6}

Segregación escolar por nivel socioeconómico para Q4 en servicios educativos estatales y no estatales en cada distrito de Lima

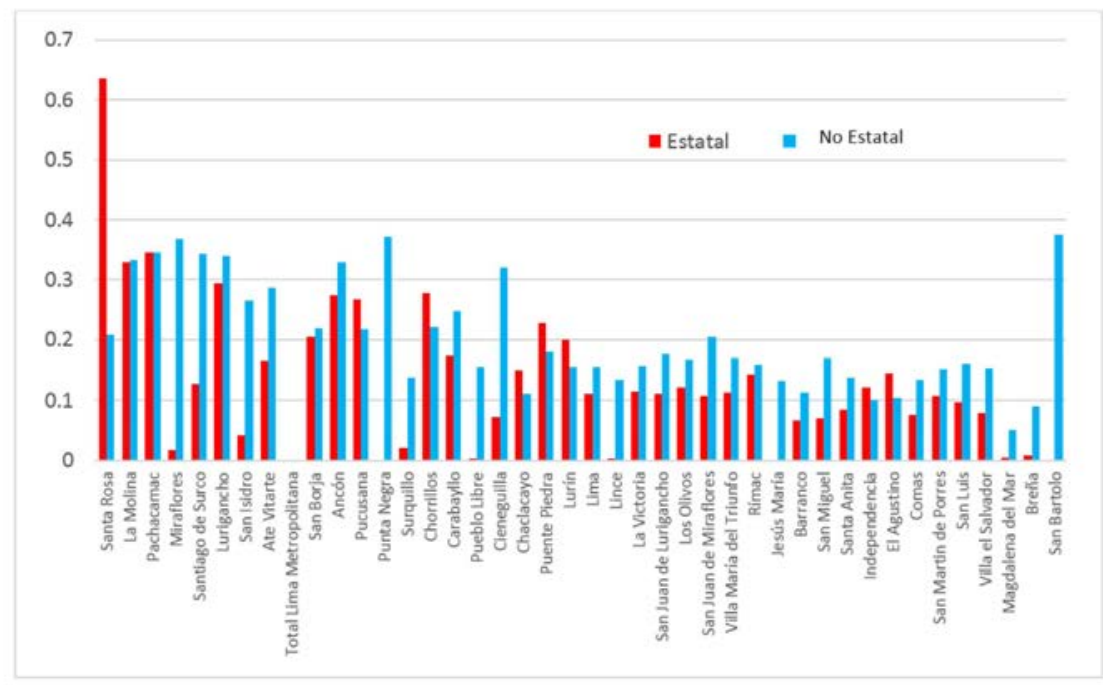

Nota: Distritos ordenados por la magnitud de la segregación total. Elaboración propia a partir de la base de datos ECE 2018 (Minedu-UMC, 2018).

Una mirada a la segregación en tres distritos de la capital: La Molina, Santa Anita e Independencia

Luego de una mirada panorámica a la segregación escolar por nivel socioeconómico en la ciudad de Lima y sus distritos, identificamos tres distritos de la capital que permiten analizar con mayor profundidad y explicar cómo se manifiesta la segregación escolar en territorios concretos. A continuación, se 
presenta cada uno de los distritos, primero, con una imagen del plano de la distribución de ingreso per cápita del hogar, según manzanas, lo que permite caracterizar visualmente la diversidad dentro de los propios distritos. Luego, se muestran las características de los servicios educativos (escuelas) que imparten educación primaria en cada uno de los distritos seleccionados. Finalmente, exponemos un gráfico por distrito con los porcentajes de estudiantes en los niveles socioeconómicos estudiados, distribuidos en cada uno de estos servicios.

\section{La Molina, un distrito rico con alta segregación escolar}

La Molina es un distrito ubicado en la zona Este de Lima. Tiene una superficie de $65,75 \mathrm{~km}^{2}$ y 140.679 habitantes según el censo del 2017. Su densidad es baja - de 2139 personas por kilómetro cuadrado-, debido a que hay grandes áreas del distrito en las que no hay viviendas por su orografía montañosa (García y Sánchez, 2019). Según el nivel de ingresos per cápita (Gráfico 7), es un distrito en el que viven las familias con los ingresos más altos del país: el $87 \%$ de su población tiene ganancias superiores a los 2400 soles mensuales, mientras que el restante $13 \%$ pertenece a los estratos medio alto y, en menor medida, medio (Carhuavilca y Sánchez, 2020). Su índice de desarrollo humano tiene un valor de 0,85 y es el distrito que ocupa el primer lugar entre todos los distritos del Perú (PNUD, 2019).

\section{Gráfico 7}

Plano del ingreso per cápita del hogar en el distrito de La Molina
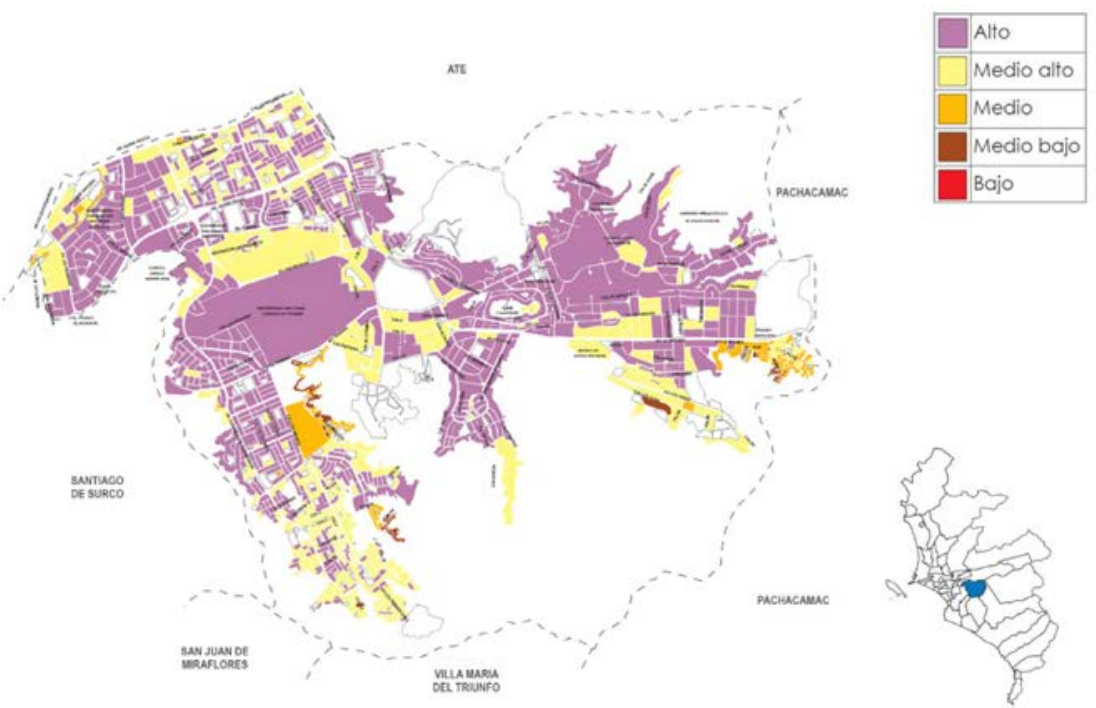
En el distrito de La Molina se encuentran 61 servicios educativos de educación primaria, de los cuales $10(16 \%)$ son estatales, con un ISE de 0,64, y el resto es no estatal (ISE de 1,53). De las 51 instituciones de gestión no estatal, el $13 \%$ tienen un costo medio - entre 151 y 350 soles -, y el $70 \%$ tienen un costo mayor a 350 soles.

La segregación escolar de La Molina es una de las más altas entre los distritos que abarca el estudio, tanto en la segregación para el 25\% de estudiantes con familias de menos recursos (Q1) $(0,53)$, como para el 25\% de los estudiantes de familias con mayor nivel socioeconómico (Q4) $(0,48)$. Así, el porcentaje de estudiantes del Q1 promedio en esas 61 escuelas es apenas un 6,9\%, mientras que el porcentaje promedio de estudiantes del Q4 asciende a 69\%.

Se observan diferencias interesantes entre las escuelas estatales y no estatales. El porcentaje promedio de estudiantes del Q1 en las escuelas estatales es del 26,4 , mientras que, en las no estatales, es de apenas un 2,2\%. Para el Q4, por su parte, el promedio de estudiantes en cada escuela es de $28,9 \%$ en escuelas estatales y de $76,9 \%$ en las instituciones no estatales. En coherencia con esas cifras, la segregación escolar en ese distrito es, para Q1, de 0,20 en escuelas estatales y de 0,72 en las no estatales; $y$, para el Q4, de 0,33 en ambos tipos de escuela.

Tabla 2

Características de los servicios educativos (escuelas) que imparten educación primaria del distrito de La Molina

\begin{tabular}{lccc}
\hline \multicolumn{1}{c}{ Características } & $\begin{array}{c}\text { Total de } \\
\text { escuelas }\end{array}$ & $\begin{array}{r}\text { Escuelas } \\
\text { estatales }\end{array}$ & $\begin{array}{c}\text { Escuelas } \\
\text { no estatales }\end{array}$ \\
\hline $\begin{array}{l}\text { Índice socioeconómico } \\
\text { (ISE) }\end{array}$ & 1,2801 & 0,6441 & 1,5261 \\
$\begin{array}{l}\text { Número de escuelas } \\
\begin{array}{l}\text { Segregación para el Q1 } \\
\text { (IH) }\end{array}\end{array}$ & 61 & 10 & 51 \\
$\begin{array}{l}\text { Segregación para el Q4 } \\
\text { (IH) }\end{array}$ & 0,4254 & 0,1990 & 0,7241 \\
$\begin{array}{l}\text { \% promedio de estudiantes } \\
\text { del Q1 }\end{array}$ & 6,19 & 0,3295 & 0,3318 \\
$\begin{array}{l}\text { \% promedio de estudiantes } \\
\text { del Q4 }\end{array}$ & 69,00 & 26,44 & 2,22 \\
\hline
\end{tabular}

Fuente: Elaboración propia a partir de la base de datos ECE 2018 (Minedu-UMC, 2018).

Una mirada más detallada de la segregación escolar en La Molina puede obtenerse a partir del análisis del nivel socioeconómico de las familias de los estudiantes que asisten a cada uno de los 61 servicios educativos que imparten 
educación primaria en el distrito. Como muestra el Gráfico 8, en que se representa cada una de las escuelas del distrito y el porcentaje de estudiantes del Q1 y del Q4 en cada una de ellas, en 45 de las 61 escuelas no hay ni un solo estudiante con familias del 25\% más pobre. En contraposición, en tres centros educativos, el porcentaje de ellos es mayor al 50\%. Queda claro, por tanto, que los pocos estudiantes que hay del Q1 se concentran en unas pocas escuelas. En coherencia con estos hallazgos, se muestra que, mientras que en 48 escuelas el $50 \%$ de los estudiantes son del cuartil más rico -incluso en 22 de ellas, más de la tercera parte de las existentes en el distrito, tienen más del 90\% de estudiantes del Q4-, en 3 de ellas este grupo no asciende al 10\%.

Así, se observa una situación muy clara, en la que los estudiantes de familias con menos recursos - que son minoría en este distrito- se escolarizan en unas pocas escuelas donde no hay estudiantes de más recursos. En paralelo, los estudiantes con familias de mayor nivel socioeconómico se escolarizan en instituciones donde no hay estudiantes con pocos recursos. Con estas cifras, se puede afirmar que el $85 \%$ de las escuelas del distrito son escuelas gueto, bien sea por su alta concentración de estudiantes del Q4 (la gran mayoría) como por su concentración de estudiantes del Q1.

\section{Gráfico 8}

Porcentaje de estudiantes del Q1 y del Q4 en nivel socioeconómico en cada uno de los servicios educativos de educación primaria del distrito de La Molina

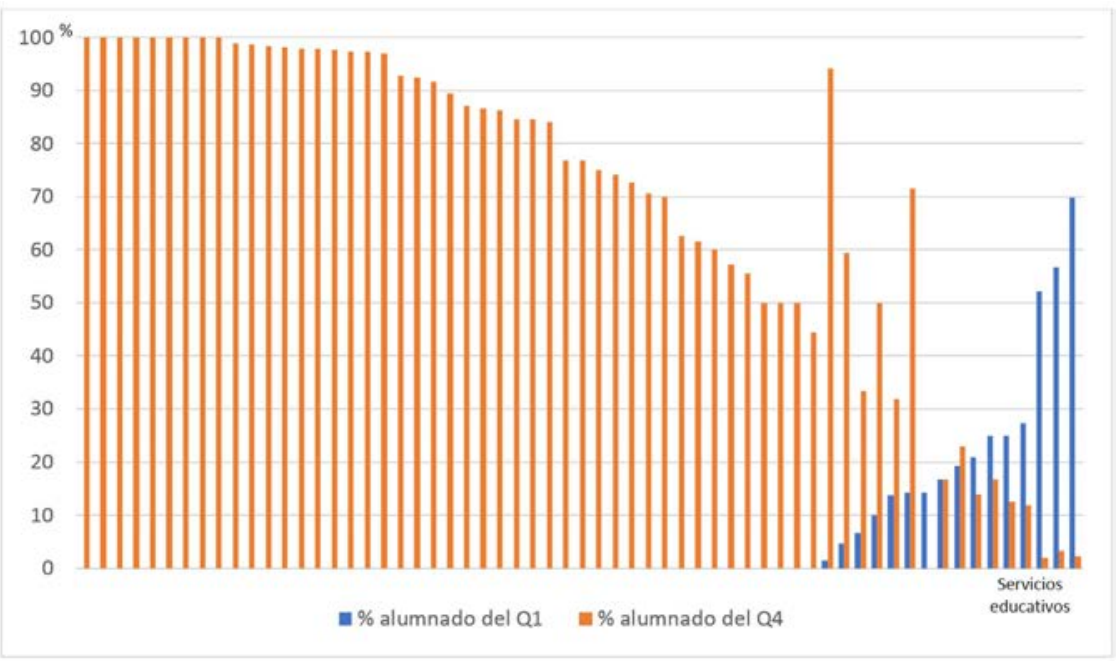

Nota: Servicios educativos del distrito ordenados por porcentaje de alumnos segregados según nivel socioeconómico (Q1 y Q4). Elaboración propia a partir de la base de datos ECE 2018 (Minedu-UMC, 2018). 
Santa Anita, un distrito de nivel socioeconómico medio con baja segregación escolar

El distrito de Santa Anita muestra una situación completamente distinta. Aunque también se encuentra situado en Lima Este, se caracteriza por el nivel socioeconómico medio de sus habitantes y es uno de los que tiene la segregación escolar más baja de la ciudad.

Santa Anita tiene una superficie de $10,69 \mathrm{~km}^{2}$ y un promedio de 196.214 habitantes según el censo del 2017, lo que implica una densidad de 18,35 personas por $\mathrm{km}^{2}, 9$ veces más que La Molina (García y Sánchez, 2019). Según el nivel de ingresos per cápita (Gráfico 9), el $72 \%$ de su población es de un estrato económico medio con ganancias que oscilan entre los 1000 y 1450 soles mensuales, mientras que $23 \%$ se ubica en el estrato medio alto (ingresos entre 1450 y 2400 soles). El restante 5\% pertenece a los estratos medio bajo y bajo (Carhuavilca y Sánchez, 2020). El distrito tiene un índice de desarrollo humano de 0,73, que lo posiciona en el número 33 entre todos los distritos del país, y 21 entre los 43 distritos de Lima Metropolitana (PNUD, 2019).

\section{Gráfico 9}

Plano del ingreso per cápita del hogar en el distrito de Santa Anita
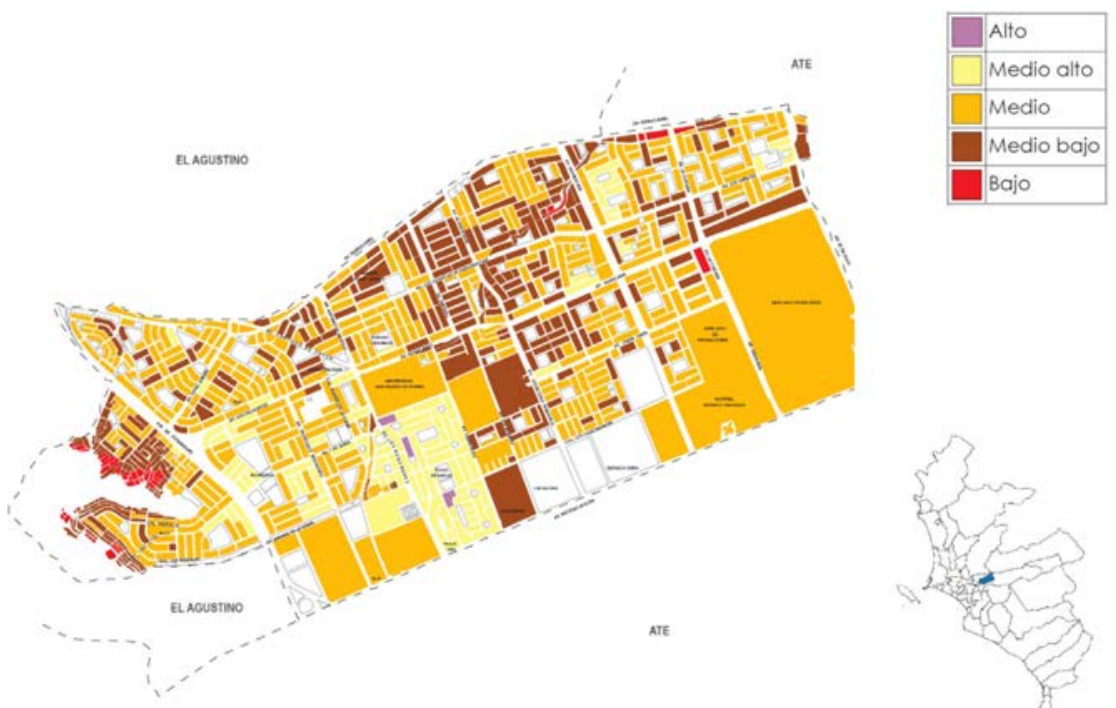

Fuente: Extraído de Carhuavilca y Sánchez (2020, p. 53). 
El distrito de Santa Anita tiene 91 servicios educativos, de los cuales 21 (23\%) son estatales y el resto son no estatales. De las 70 instituciones de gestión no estatal, el $3 \%$ son de un costo bajo (hasta 150 soles de pensión), 68\% de un costo mediano (entre 151 y 350 soles) y $6 \%$ de costo más alto (más de 350 soles).

La segregación escolar en el distrito de Santa Anita es globalmente baja $(0,15)$ para los estudiantes de menos recursos (Q1) y ligeramente superior $(0,21)$ para los estudiantes de mayores recursos (Q4). De esta manera, el porcentaje de estudiantes de Q1 promedio es $17 \%$ y el porcentaje promedio de estudiantes del Q4 es 19\%.

Sobre las diferencias entre las escuelas estatales y no estatales, se identifica que el porcentaje promedio de estudiantes del Q1 en las escuelas estatales es del $32,3 \%$, mientras que en las no estatales es de $12,8 \%$. Para el Q4, el promedio de estudiantes en escuelas estatales es de 5,1\% y de 23,3\% en las no estatales. En concordancia con esas cifras, la segregación escolar en este distrito es, para Q1, de 0,02 en escuelas estatales y de 0,19 en las no estatales; y, para el Q4, de 0,32 en las escuelas estatales y de 12,8 en las no estatales.

Tabla 3

Características de los servicios educativos (escuelas) del distrito de Santa Anita

\begin{tabular}{lccc}
\hline \multicolumn{1}{c}{ Distrito } & $\begin{array}{c}\text { Total de } \\
\text { escuelas }\end{array}$ & $\begin{array}{c}\text { Escuelas } \\
\text { estatales }\end{array}$ & $\begin{array}{c}\text { Escuelas } \\
\text { no } \\
\text { estatales }\end{array}$ \\
\hline $\begin{array}{l}\text { Índice socioeconómico promedio de } \\
\text { las familias de los estudiantes }\end{array}$ & 0,6246 & 0,3236 & 0,7757 \\
$\begin{array}{l}\text { Número de escuelas } \\
\text { Segregación para el Q1 }\end{array}$ & 91 & 21 & 70 \\
$\begin{array}{l}\text { Segregación para el Q4 } \\
\begin{array}{l}\text { Porcentaje promedio de estudiantes } \\
\text { del Q1 }\end{array}\end{array}$ & 0,1451 & 0,0168 & 0,1901 \\
$\begin{array}{l}\text { Porcentaje promedio de estudiantes } \\
\text { del Q4 }\end{array}$ & 17,28 & 32,27 & 12,79 \\
\hline
\end{tabular}

Fuente: Elaboración propia a partir de la base de datos ECE 2018 (Minedu-UMC, 2018).

Como se muestra en el gráfico 10, en 15 de los 91 servicios educativos del nivel primaria en Santa Anita, no hay ni un solo estudiante con familias pertenecientes al 25\% más pobre, mientras que, en 4 centros educativos, el porcentaje de ellos corresponde a más del 50\%. De igual manera, en 8 escuelas del distrito no hay ningún estudiante del Q4, mientras que en 7 de ellas hay más de 50\%. Es decir, frente a lo observado en el distrito La Molina, en el distrito 
de Santa Anita hay pocas escuelas donde no hay estudiantes del Q1, así como del Q4, y son pocas las que tienen el 100\% de niños y niñas del Q1 o Q4. De esta forma, sin dejar de haber segregación, esta es mucho más moderada: los estudiantes se encuentran más repartidos en las escuelas en Santa Anita que en el caso de La Molina.

\section{Gráfico 10}

Porcentaje de estudiantes del Q1 y del Q4 en nivel socioeconómico en cada uno de los servicios educativos de educación primaria del distrito de Santa Anita

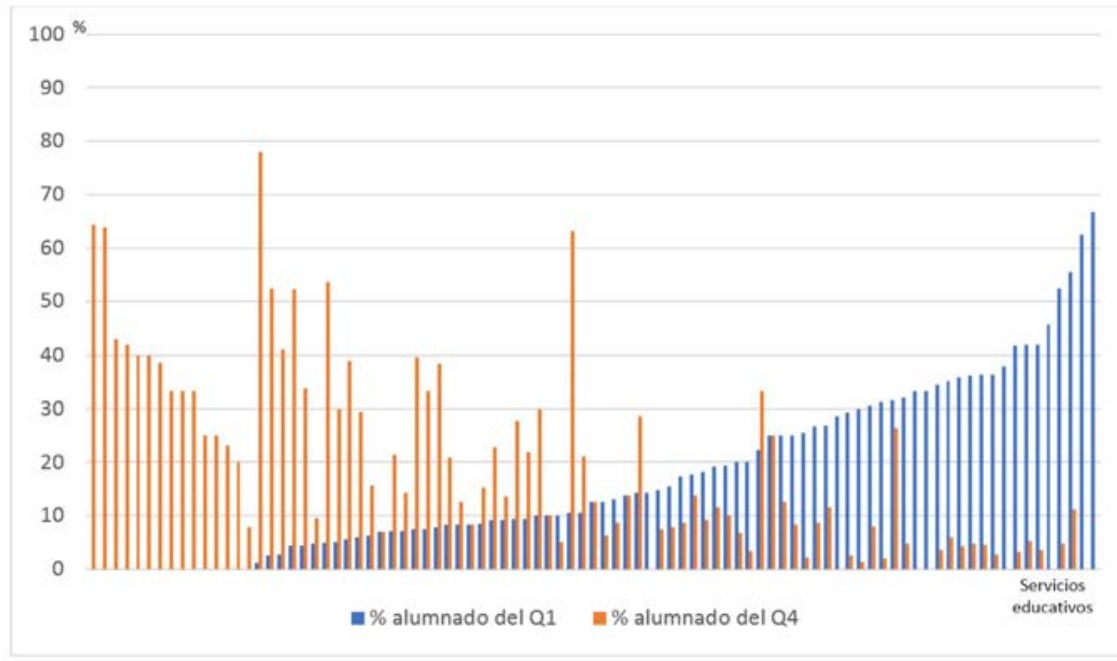

Nota: Servicios educativos del distrito ordenados por porcentaje de alumnos segregados según nivel socioeconómico (Q1 y Q4). Elaboración propia a partir de la base de datos ECE 2018 (Minedu-UMC, 2018).

\section{Independencia, un distrito pobre con baja segregación escolar}

El tercer distrito estudiado es Independencia. Este caso fue seleccionado, debido a que una mayoría de su población es de nivel económico bajo o mediobajo, y a que existe bajos índices de segregación escolar.

Independencia está ubicado en la zona norte de Lima, tiene una superficie de 14,56 $\mathrm{km}^{2}$ y un promedio de 211.360 habitantes según el censo del 2017. Así, su densidad es alta (14,51 de personas por kilómetro cuadrado), y ligeramente más baja a la de Santa Anita (García y Sánchez, 2019). Según el nivel de ingresos per cápita (Gráfico 11), el 59\% de la población es de un estrato económico bajo o muy bajo, con ingresos inferiores a 900 soles mensuales por hogar, y el $41 \%$ de su población es de un estrato económico medio, con ganancias entre los 1000 y 1450 soles mensuales (Carhuavilca y Sánchez, 
2020). El distrito tiene un índice de desarrollo humano con un valor de 0,69, y ocupa el lugar 65 de todos los distritos del Perú, y el 30 entre los 43 distritos de Lima (PNUD, 2019).

\section{Gráfico 11}

Plano del ingreso per cápita del hogar en el distrito de Independencia
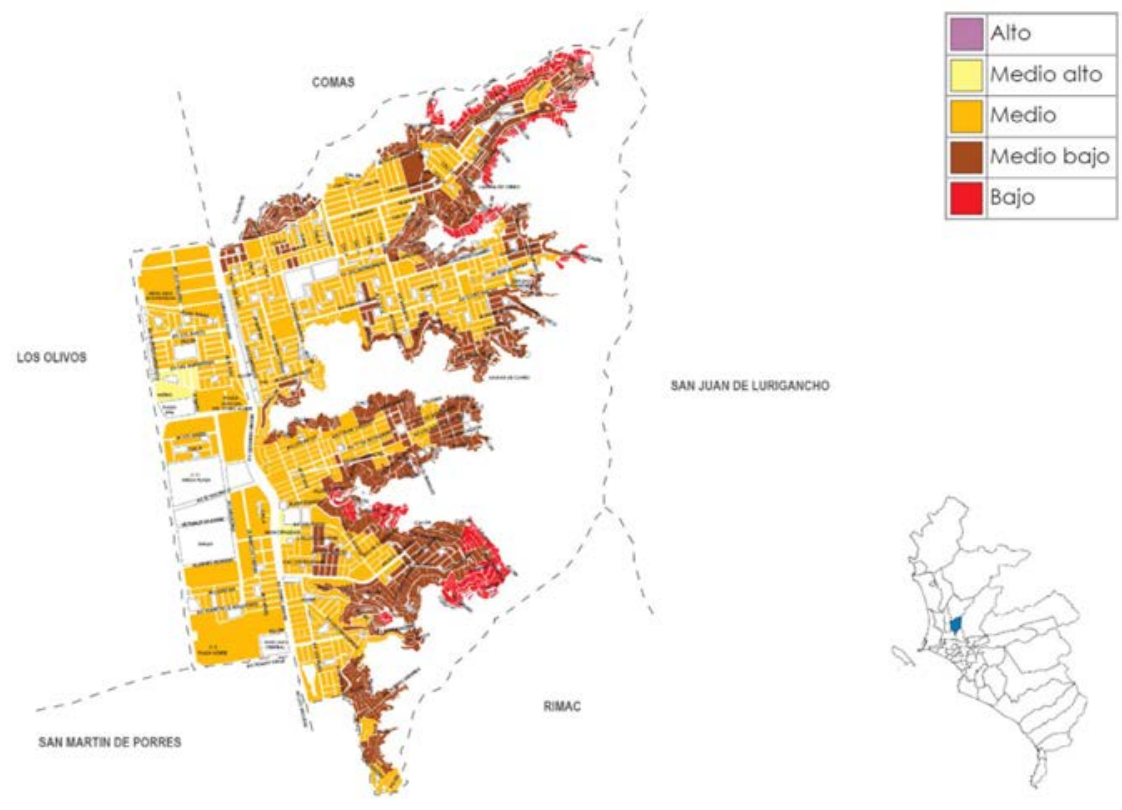

Fuente: Extraído de Carhuavilca y Sánchez (2020, p. 28).

Independencia tiene 74 servicios de educación primaria, de los cuales 23 (31\%) son estatales, mientras que los 51 restantes son no estatales. De los últimos, el $19 \%$ son de costo bajo (hasta 150 soles de pensión), 47\% de costo mediano (entre 151 y 350 soles) y solo el $1 \%$ es de costo más alto (más de 350 soles).

La segregación escolar en el distrito de Independencia es globalmente baja $(0,13)$ para los estudiantes de menos recursos $(\mathrm{Q} 1)$ y ligeramente superior $(0,19)$ para los estudiantes de mayores recursos $(\mathrm{Q} 4)$. Así, el porcentaje de estudiantes de Q1 promedio es 19,6\%, que corresponde al mismo porcentaje promedio de estudiantes del Q4.

Con respecto a las diferencias entre las escuelas estatales y no estatales, se muestra que el porcentaje promedio de estudiantes del Q1 en las escuelas públicas es del 38,4\%, mientras que, en las no estatales, corresponde al $11,2 \%$. Para el Q4, el promedio de estudiantes en escuelas estatales es de 5,1\% y del $26,2 \%$ en las no estatales. En coherencia con esas cifras, la segregación escolar 
en el distrito de Independencia es, para Q1, de 0,05 en escuelas estatales y de 0,14 en las no estatales; $y$, para Q4, de 0,12 en las escuelas estatales y de 0,1 en las no estatales.

Tabla 4

Características de los servicios educativos (escuelas) del distrito de Independencia

\begin{tabular}{lccc}
\multicolumn{1}{c}{ Distrito } & $\begin{array}{c}\text { Total de } \\
\text { escuelas }\end{array}$ & $\begin{array}{c}\text { Escuelas } \\
\text { estatales }\end{array}$ & $\begin{array}{c}\text { Escuelas } \\
\text { no estatales }\end{array}$ \\
\hline Índice socioeconómico de los estudiantes (ISE) & 0,4405 & 0,2099 & 0,8276 \\
Número de escuelas & 74 & 23 & 51 \\
Segregación para el Q1 & 0,1255 & 0,0458 & 0,1435 \\
Segregación para el Q4 & 0,1918 & 0,1220 & 0,0995 \\
Porcentaje promedio de estudiantes del Q1 & 19,64 & 38,43 & 11,16 \\
Porcentaje promedio de estudiantes del Q4 & 19,65 & 5,09 & 26,22 \\
\hline
\end{tabular}

Fuente: Elaboración propia a partir de la base de datos ECE 2018 (Minedu-UMC, 2018).

El gráfico 12 muestra el porcentaje de estudiantes del Q1 y del Q4 en cada uno de los 74 servicios educativos (escuelas) del distrito de Independencia. En este caso, puede observarse que, en una docena de escuelas, no hay ni un solo estudiante con familias del $25 \%$ más pobre, mientras que, en 5 centros educativos, el porcentaje de ellos corresponde a más del 50\%. Por su parte, en 10 escuelas no hay ningún estudiante del Q4, y, en seis, estos superan el 50\%. Incluso, existe una escuela en la que todo el alumnado pertenece al Q4.

El perfil del gráfico se asemeja mucho al panorama de Santa Anita y se diferencia del caso de La Molina. Aunque existe segregación, se observa que hay un mayor reparto de los estudiantes tanto del Q1 como del Q4 en todas las escuelas. En esa medida, el número de instituciones educativas que podemos considerar gueto, tanto para el Q1 como para el Q4, es muy escaso. 


\section{Gráfico 12}

Porcentaje de estudiantes del Q1 y del Q4 en cada uno de los servicios educativos de educación primaria del distrito de Independencia

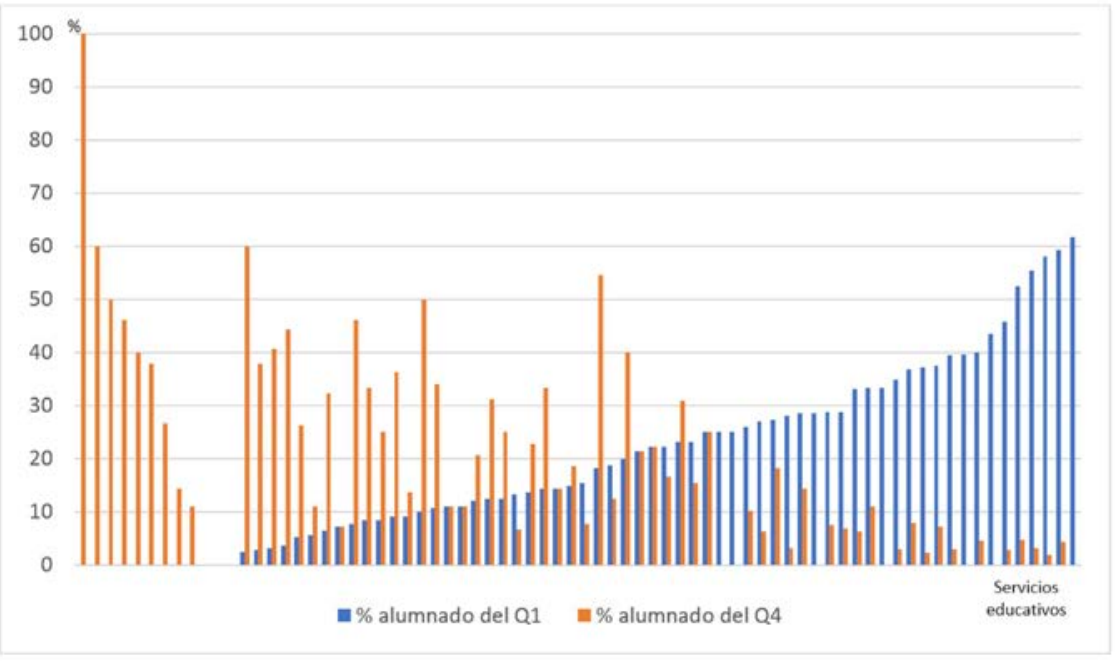

Nota: Servicios educativos del distrito ordenados por porcentaje de alumnos segregados según nivel socioeconómico (Q1 y Q4). Elaboración propia a partir de la base de datos ECE 2018 (Minedu-UMC, 2018).

\section{Discusión y conclusiones}

Esta investigación muestra la fuerte relación entre distribución socioespacial y segregación escolar en Lima Metropolitana. Así, por ejemplo, se ha visto que los distritos de Lima Centro tienen un comportamiento en segregación escolar bastante diferente del resto. De la misma manera, el estudio aporta evidencia de cómo el nivel económico de cada distrito determina, en gran medida, la segregación escolar en el caso de las poblaciones más pobres y, en menor medida, la segregación de aquellos que son más ricos.

Sin embargo, se dan excepciones a esa pauta general. Así, hemos identificado diferentes distritos que, a pesar de su situación de riqueza, consiguen que haya una baja segregación en sus escuelas. Es decir, en términos generales y respondiendo al objetivo planteado en esta investigación, el estudio da cuenta de que, en la ciudad de Lima Metropolitana, las desigualdades socioespaciales inciden de forma clara en la segregación escolar.

Escasa literatura, y aún menor en el ámbito de América Latina, ha abordado el problema de investigación tal y como se ha realizado en este estudio, por lo cual es difícil desarrollar una discusión de los resultados. Los resultados parciales encontrados en esta investigación coinciden con los hallazgos de otros 
autores. Así, los niveles de segregación escolar identificados en la educación primaria para Lima Metropolitana concuerdan con los resultados de Murillo y Carrillo (2020a), con la diferencia de que dichos investigadores utilizaron el índice de Gorard, en contraste con el presente estudio, que empleó el índice de Hutchens. También, se observa un paralelo con los análisis del importante rol de la educación privada en la segregación - por ejemplo, Murillo y Martínez-Garrido (2017b) o el de Murillo et al. (2020) para toda América Latina-, marco en el cual los resultados de Perú son especialmente altos.

Resulta especialmente importante el diálogo con investigaciones que han abordado las desigualdades socioespaciales y su relación con la segregación escolar en ámbitos locales. Sirviéndose de información distrital, este trabajo va en la línea de algunos interesantes estudios enfocados en ciudades específicas que muestran que la desigualdad espacial incide en la segregación escolar. Ejemplo de ello se observa en los resultados en la investigación de Bonal et al. (2019) sobre la segregación escolar por origen nacional en la ciudad de Barcelona o en Santos y Elacqua (2016) para el caso de escuelas del Gran Santiago. En ambos, se observa que la segregación escolar trasciende el efecto de la segregación residencial subyacente en ambas ciudades. Planteamientos análogos destacan en los hallazgos de Rujas et al. (2020) para Madrid, o Bartholo y da Costa (2018) para cuatro ciudades de Brasil - Río de Janeiro, São Paulo, Belo Horizonte y Curitiba-. En el trabajo de Bonal et al. (2019) se muestra, también, que la oferta de enseñanza privada subvencionada en los distritos es el principal factor que impulsa tanto la segregación escolar como el aislamiento, especialmente, en aquellos distritos con una alta concentración de alumnos extranjeros. Si bien aún no hay suficiente evidencia en la región, esta investigación muestra claramente que la segregación escolar es un tema que va más allá de las diferencias socioespaciales generadas por la segregación residencial.

Estos hallazgos nos llevan a plantear algunas recomendaciones que nos parecen relevantes para la política educativa, dada la necesidad y la urgencia de enfrentar con firmeza y determinación el fenómeno de la segregación escolar en el país. En primer lugar, se sugiere tener en cuenta las desigualdades socioespaciales para hacer políticas diferenciadas en función de las zonas y de los colectivos. Es decir, es preciso que las medidas generales contra la segregación para todo el país se acompañen de acciones diferenciadas para entornos locales que respeten sus características y sus problemáticas.

En segundo lugar, es importante y necesario combatir la segregación escolar de los hijos de las familias con mayor nivel socioeconómico. Tanto las escuelas conformadas por alumnado vulnerable como aquellas en que todos los y las estudiantes proceden de familias con mayores recursos se constituyen como un gueto. Ambas son perjudiciales para la calidad del sistema educativo y para la conformación de una sociedad más justa, en las que el derecho a la educación no sea vulnerado. Esta investigación invita a mirar y combatir tanto la segregación escolar de los hijos de las familias más vulnerables como la de los hijos de las familias más acomodadas. 
En tercer lugar, una vez más, se ha visto la crucial contribución de las escuelas no estatales en la segregación escolar. De esta forma, se recomienda revisar y mejorar la regulación de la oferta de la educación privada, especialmente aquella relacionada a las escuelas de bajo costo, que atienden a los hijos de las familias de menores recursos.

Esta investigación, como cualquier otra, tiene fortalezas y limitaciones que deben ser visibilizadas. Con respecto a su fortaleza, el estudio aborda, con datos censales que el Ministerio de Educación pone a disposición de la comunidad académica, el estudio de la segregación escolar con una mirada a profundidad en una ciudad. En este caso, se trata de Lima Metropolitana, una de las ciudades con mayor nivel de segregación de la región.

En cuanto a sus limitaciones, se reconoce que el uso del ECE como fuente principal de información implica ciertas restricciones. Un estudio más exhaustivo requeriría de más datos. Así, por ejemplo, contar con los datos de todos los estudiantes de la escuela $-\mathrm{y}$ no solo un grado- con información del lugar de residencia de los estudiantes - y no solo del distrito de la escuela- permitiría aumentar la profundidad de la investigación.

Finalmente, es necesario plantear futuras investigaciones sobre el tema. Sería importante profundizar en los casos que consiguen tener una baja segregación y aprender de ellos: qué es lo que está funcionando y que no, qué es lo que es replicable o adaptable para contextos similares. Es decir, necesitamos de una investigación aplicada y contextualizada que nos dé claras pautas para cambiar esta situación que incrementa la desigualdad social, y que tiene una fuerte repercusión negativa en la calidad y en la equidad del sistema educativo peruano. 


\section{Referencias bibliográficas}

Alegre, M. (2010). Casi-mercados, segregación escolar y desigualdad educativa: una trilogía con final abierto. Educacao \& Sociedade, 41(114), 1157-1178. https://doi.org/10.1590/S0101-73302010000400006

Balarin, M. y Escudero, A. (2018). The ungoverned education market and the deepening of socio-economic school segregation in Peru. En X. Bonal y C. Bellei (Eds.), Understanding School Segregation. Patterns, Causes and Consequences of Spatial Inequalities in Education (pp. 179-199). Bloomsbury Academic.

Bartholo, T. y Costa, M. (2018). Patterns of School Segregation in Brazil: Inequalities and Education Policy. En X. Bonal y C. Bellei (Eds.), Understanding School Segregation. Patterns, Causes and Consequences of Spatial Inequalities in Education (pp. 179-199). Bloomsbury Academic.

Bellei, C. (2013). El estudio de la segregación económica y académica de la educación chilena. Estudios Pedagógicos, 39(1), 325-345. https://doi. org/10.4067/S0718-07052013000100019

Bellei, C., Contreras, M., Canales, M. y Orellana, V. (2018). The Production of Socio-Economic Segregation in Chilean Education: School Choice, Social Class and Market Dynamics. En X. Bonal y C. Bellei (Eds.), Understanding school segregation. patterns, causes and consequences of spatial inequalities in education (pp. 221-240). Bloomsbury Academic.

Benavides, M., León, J. y Etesse, M. (2014). Desigualdades educativas y segregación en el sistema educativo peruano: una mirada comparativa de las pruebas PISA 2000 y 2009. Grade.

Bonal, X., Zancajo, A. y Scandurra, R. (2019). Residential Segregation and School Segregation of Foreign Students in Barcelona. Urban Studies, 56(15), 3251-3273. https://doi.org/10.1177/0042098019863662

Burke, M. A. y Sass, T. R. (2013). Classroom Peer Effects and Student Achievement. Journal of Labor Economics, 31(1), 51-82. https://doi. org/10.2139/ssrn. 1260882

Carrillo, S., Salazar, V. y Leandro, S. (2019). Jóvenes y educación en Lima Metropolitana y Callao. Documento de Trabajo, 259. Serie Educación 19. Instituto de Estudios Peruanos.

Carhuavilca, D. y Sánchez, A. (2020). Planos estratificados de Lima Metropolitana a nivel de manzanas 2020. Instituto Nacional de Estadística e Informática.

Cuenca, R. (2013). La escuela pública en Lima Metropolitana. ¿ Una institución en extinción? Revista Peruana de Investigación Educativa, 1(5), 73-98.

Cuenca, R. y Urrutia, C. (2019). Explorando las brechas de desigualdad educativa en el Perú. Revista Mexicana de Investigación Educativa RMIE, 24 (81), 431-461. 
Cueto, S. (2021). Las políticas y programas que no se diseñan explícitamente para promover equidad aumentan la inequidad. Una hipótesis para el Perú. En C. Guadalupe (ed.). La educación peruana más allá del Bicentenario: nuevos rumbos (pp.417-442). Universidad del Pacífico.

Cueto, S., León, J. y Miranda, A. (2016). Classroom Composition and Its Association With Students' Achievement and Socioemotional Characteristics in Peru. Assessment in Education: Principles, Policy \& Practice, 23(1), 126-148. https://doi.org/10.1080/0969594X.2015.1105783

Durlauf, S. N. (2006). Groups, Social Influences, And Inequality: A Memberships Theory Perspective on Poverty Traps. En S. Bowles, S. Durlauf y K. Hoff (Eds.), Poverty traps (pp. 79-101). Princeton University Press.

Dupriez, V. (2010). Methods of Grouping Learners at School. Unesco.

Elacqua, G. (2009). The impact of school choice and public policy on segregation: Evidence from Chile. Centro de Políticas Comparadas de Educación Universidad Diego Portales.

García-Huidobro, J. E. (2007). Desigualdad educativa y segmentación del sistema escolar. Consideraciones a partir del caso chileno. Pensamiento Educativo, 40(1), 65-86.

García, J. y Sánchez, A. (2019). Compendio estadístico: Provincia de Lima 2019. Instituto Nacional de Estadística e Informática.

Guadalupe, C., León, J., Rodríguez, J. y Vargas, S. (2017). Estado de la educación en el Perú. Análisis y perspectivas de la educación básica. Grade.

Hutchens, R. (2004). One Measure of Segregation. International Economic Review, 45(2), 555-578. https://doi.org/10.1111/j.1468-2354.2004.00136.x

Instituto Nacional de Estadística e Informática [INEI] (2020). Mapa de pobreza monetaria provincial y distrital 2018. INEI.

Krüger, N. (2019). La segregación por nivel socioeconómico como dimensión de la exclusión educativa: 15 años de evolución en América Latina. Archivos Analíticos de Políticas Educativas, 27(8), 1-37. https://doi.org/10.14507/ epaa.27.3577

Martínez, L. y Ferrer, A. (2018). Mézclate conmigo. De la segregación socioeconómica a la educación inclusiva. Save the Children.

Ministerio de Educación (2018a). Desafíos en la medición y el análisis del estatus socioeconómico de los estudiantes peruanos. Oficina de Medición de la Calidad de los Aprendizajes del Ministerio de Educación del Perú.

Ministerio de Educación (2018b). Tipología y caracterización de las escuelas privadas en el Perú. Serie Estudios Breves 3. Ministerio de Educación, Oficina de Medición de la Calidad de los Aprendizajes.

Ministerio de Educación - Oficina de Medición de la Calidad de los Aprendizajes [Minedu-UMC] (2018). Evaluación Censal de Estudios 2018 [base de datos]. Recuperado de http://umc.minedu.gob.pe/resultados-generales-en-el-tiempo/ 
Miranda, L. (2021). Tensiones entre segregación escolar y desarrollo ciudadano. En C. Guadalupe (Ed.). La educación peruana más allá del Bicentenario: nuevos rumbos (pp.469-493). Universidad del Pacífico.

Murillo, F. J. (2016). Midiendo la segregación escolar en América Latina. Un análisis metodológico utilizando el TERCE. Reice. Revista Iberoamericana sobre Calidad, Eficacia y Cambio en Educación, 14(4), 33-60. https://doi. org/10.15366/reice2016.14.4.002

Murillo, F. J. y Carrillo, S. (2020a). Segregación escolar por nivel socioeconómico en educación secundaria en Perú y sus regiones. Revista Peruana de Investigación Educativa, 1(12), 7-32. https://doi.org/10.34236/rpie.v12i12.130

Murillo, F. J. y Carrillo, S. (2020b). Una panorámica de la segregación escolar por nivel socioeconómico en Educación Primaria en Perú y sus regiones. Revista Argumentos, 1(1), 7-31. https://doi.org/10.46476/ra.vi1.9

Murillo, F. J. y Carrillo, S. (2021). Incidencia de la segregación escolar por nivel socioeconómico en el rendimiento académico. Un estudio desde Perú. Archivos Analíticos de Políticas Educativas, 29(49), 1-24. https://doi.org/10.14507/ epaa.29.5129

Murillo, F. J., Duk, C. y Martínez-Garrido, C. (2018). Evolución de la segregación socioeconómica de las escuelas de América Latina. Estudios Pedagógicos, 44(1), 157-179. https://doi.org/10.4067/S0718-07052018000100157

Murillo, F. J. y Martínez-Garrido, C. (2017a). Estimación de la magnitud de la segregación escolar en América Latina. Magis. Revista Internacional de Investigación Educativa, 9(19), 11-30. https://doi.org/10.11144/javeriana.m919.emse

Murillo, F. J. y Martínez-Garrido, C. (2017b). Segregación en las escuelas públicas y privadas en América Latina. Educação \& Sociedade, 38(140), 727750. https://doi.org/10.1590/es0101-73302017167714

Murillo, F. J., Martínez-Garrido, C. y Graña, R. (2020). Escuelas públicas para pobres, escuelas privadas para ricos: relación entre educación privada y segregación escolar de carácter socio-económico en América Latina. RUNAE, $5,11-22$.

Organisation for Economic Co-operation and Development [OECD] (2019). Balancing School Choice and Equity. An International Perspective based on PISA. OECD Publishing.

Pereyra, O. (2006). Forma urbana y segregación residencial en Lima. Debates en Sociología, 31, 69-106.

Programa de las Naciones Unidas para el Desarrollo [PNUD] (2019). El reto de la igualdad. Una lectura de las dinámicas territoriales en el Perú. PNUD.

Roca, M. (2012). Segregación social: distribución y construcción espacial en Lima Metropolitana. Espacio y Desarrollo, 24, 119-134.

Rujas, J., Prieto, M. y Rogero-García, J. (2020). Desigualdades socioespaciales en la educación secundaria postobligatoria. El caso de Madrid. REICE. Revista Iberoamericana sobre Calidad, Eficacia y Cambio en Educación, 18(4), 241-267. https://doi.org/10.15366/reice2020.18.4.010 
Sabatini, F. (1999). Tendencias de la segregación residencial urbana en Latinoamérica: Reflexiones a partir del caso de Santiago de Chile. Universidad de Uppsala.

Santos, H. y Elacqua, G. (2016). Segregación socioeconómica escolar en Chile: elección de la escuela por los padres y un análisis contrafactual teórico. Revista CEPAL, 119, 133-148.

Valenzuela, J. P., Bellei, C. y Ríos, D. L. (2014). Socioeconomic School Segregation in a Market-oriented Educational System. The Case of Chile. Journal of Education Policy, 29(2), 217-241. https://doi.org/10.1080/02680939.2013.80 6995

Vazquez, E. (2016). Segregación escolar por nivel socioeconómico. Midiendo el fenómeno y explorando sus determinantes. Económica, 62, 121-184.

Wiese, C., Miyashiro, J. y Marcés, R. (2016). Desigualdad urbana en Lima Metropolitana. En W. Jungbluth (Comp.), Perú hoy. Desigualdad y desarrollo (pp. 333-368). Centro de Estudios y Promoción del Desarrollo. 


\section{Anexo}

Tabla 5

Segregación escolar en educación primaria en Lima Metropolitana en cada distrito. Índice H para Q1 y Q4

\begin{tabular}{|c|c|c|c|c|c|c|}
\hline \multirow[b]{2}{*}{ Distrito } & \multicolumn{3}{|c|}{ Q1 } & \multicolumn{3}{|c|}{ Q4 } \\
\hline & Total & Estatal & $\begin{array}{c}\text { No } \\
\text { estatal }\end{array}$ & Total & Estatal & $\begin{array}{c}\text { No } \\
\text { estatal }\end{array}$ \\
\hline Ancón & 0,1715 & 0,1348 & 0,1725 & 0,3255 & 0,2748 & 0,3289 \\
\hline Ate Vitarte & 0,1845 & 0,0507 & 0,2147 & 0,3373 & 0,1651 & 0,2858 \\
\hline Barranco & 0,2492 & 0,0302 & 0,6085 & 0,2073 & 0,0660 & 0,1132 \\
\hline Breña & 0,2746 & 0,0321 & 0,3740 & 0,1566 & 0,0083 & 0,0900 \\
\hline Carabayllo & 0,1963 & 0,0763 & 0,2056 & 0,3004 & 0,1728 & 0,2475 \\
\hline Chaclacayo & 0,2054 & 0,0182 & 0,3171 & 0,2694 & 0,1498 & 0,1102 \\
\hline Chorrillos & 0,2665 & 0,1196 & 0,3617 & 0,3011 & 0,2768 & 0,2217 \\
\hline Cieneguilla & 0,0925 & 0,0202 & 0,1297 & 0,2714 & 0,0727 & 0,3206 \\
\hline Comas & 0,1790 & 0,0586 & 0,2479 & 0,1871 & 0,0748 & 0,1337 \\
\hline El Agustino & 0,1534 & 0,0733 & 0,1945 & 0,1894 & 0,1444 & 0,1045 \\
\hline Independencia & 0,1255 & 0,0458 & 0,1435 & 0,1918 & 0,1220 & 0,0995 \\
\hline Jesús María & 0,4432 & 0,0073 & 0,5939 & 0,2116 & 0,0000 & 0,1324 \\
\hline La Molina & 0,5254 & 0,1990 & 0,7241 & 0,4847 & 0,3295 & 0,3318 \\
\hline La Victoria & 0,2471 & 0,0691 & 0,3344 & 0,2255 & 0,1141 & 0,1577 \\
\hline Lima & 0,2324 & 0,0708 & 0,3021 & 0,2328 & 0,1108 & 0,1544 \\
\hline Lince & 0,3055 & 0,0123 & 0,5725 & 0,2268 & 0,0023 & 0,1348 \\
\hline Los Olivos & 0,2194 & 0,0561 & 0,3264 & 0,2202 & 0,1222 & 0,1669 \\
\hline Lurigancho & 0,2149 & 0,1305 & 0,2373 & 0,3907 & 0,2931 & 0,3398 \\
\hline Lurín & 0,1441 & 0,0843 & 0,1008 & 0,2498 & 0,1998 & 0,1552 \\
\hline Magdalena del Mar & 0,3589 & 0,0220 & 0,6671 & 0,1738 & 0,0049 & 0,0505 \\
\hline Miraflores & 0,5179 & 0,0386 & 0,7134 & 0,4352 & 0,0176 & 0,3684 \\
\hline Pachacamac & 0,1551 & 0,0234 & 0,1698 & 0,4635 & 0,3454 & 0,3446 \\
\hline Pucusana & 0,0945 & 0,0144 & 0,0650 & 0,3133 & 0,2669 & 0,2175 \\
\hline Pueblo Libre & 0,3988 & 0,0427 & 0,8231 & 0,2845 & 0,0028 & 0,1554 \\
\hline Puente Piedra & 0,1259 & 0,0358 & 0,1125 & 0,2582 & 0,2288 & 0,1798 \\
\hline Punta Negra & 0,2247 & 0,0000 & 0,3360 & 0,3034 & 0,0000 & 0,3707 \\
\hline Rímac & 0,2243 & 0,0920 & 0,3569 & 0,2127 & 0,1427 & 0,1582 \\
\hline San Bartolo & 0,0798 & 0,0000 & 0,1036 & 0,1096 & 0,0000 & 0,3745 \\
\hline
\end{tabular}




\begin{tabular}{lllllll} 
San Borja & 0,4470 & 0,1626 & 0,8150 & 0,3306 & 0,2043 & 0,2186 \\
San Isidro & 0,6217 & 0,0139 & 0,6884 & 0,3757 & 0,0419 & 0,2644 \\
San Juan de Lurigancho & 0,1679 & 0,0537 & 0,2325 & 0,2237 & 0,1115 & 0,1766 \\
San Juan de Miraflores & 0,1989 & 0,0735 & 0,2913 & 0,2187 & 0,1079 & 0,2056 \\
San Luis & 0,1507 & 0,0776 & 0,2974 & 0,1839 & 0,0967 & 0,1600 \\
San Martin de Porres & 0,2162 & 0,0573 & 0,2917 & 0,1854 & 0,1065 & 0,1513 \\
San Miguel & 0,5054 & 0,1924 & 0,5660 & 0,2053 & 0,0698 & 0,1698 \\
Santa Anita & 0,1451 & 0,0168 & 0,1901 & 0,2051 & 0,0845 & 0,1373 \\
Santa Rosa & 0,4019 & 0,3165 & 0,4104 & 0,4882 & 0,6362 & 0,2090 \\
Santiago de Surco & 0,5401 & 0,1823 & 0,7477 & 0,3922 & 0,1272 & 0,3427 \\
Surquillo & 0,2993 & 0,0182 & 0,6956 & 0,3017 & 0,0204 & 0,1370 \\
Villa el Salvador & 0,1308 & 0,0450 & 0,1829 & 0,1811 & 0,0788 & 0,1534 \\
Villa María del Triunfo & 0,1543 & 0,0404 & 0,1805 & 0,2164 & 0,1126 & 0,1699 \\
\hline Total Lima Metropolitana & 0,2520 & & & 0,3327 & & \\
\hline
\end{tabular}

Fuente: Elaboración propia a partir de la base de datos ECE 2018 (Minedu-UMC, 2018). 